\title{
The Influence of the Chinese Intuitional and Synthetic Thought Patterns on English Learning
}

\author{
Liwei Sun \\ School of Arts and Science, Jilin Agricultural Science and Technology College, Jilin 132101
}

Keywords: Influence, Intuitional and Synthetic Thought Patterns, English learning.

\begin{abstract}
This paper tends to discuss the influence of the Chinese intuitional and synthetic thought patterns on English learning. Language is the mirror of thinking. How we think will definitely influences the way we speak. Chinese and Western thought patterns are different, which contributes to the different language system. Therefore, in order to master a target language, it is necessary to study the factors influence the mastery of that language, thought patterns in particular.
\end{abstract}

\section{Introduction}

Language is the reflection of the native culture of those who speak it. Chinese culture has exerted a great influence on the true adaptation of a foreign language more effectively and efficiently. Therefore, in order to learn the target language well, it is necessary for us to consider the influence of Chinese culture, Chinese thought patterns in particular, on English learning. To achieve this objective, this paper attempts to study the influences of Chinese intuitional and synthetic thought patterns on English learning through a corpus based study and summarizes some common errors easily appeared in the process of English learning.

\section{Chinese thought patterns}

\section{Intuitional thought pattern}

Reviewing traditional Chinese philosophy represented by Confucius, Taoist, Moh, etc., wecan see that the Chinese tend to connect the nature and the principle of the objects with the knowledge and experience they already have and they are accustomed to set up concrete things or objects to explain the philosophical theories and ideas, and there are few abstract nouns in their philosophical works. Take the "Five Elements" for example. Originally the five Chinese characters representing "Five Element" means "metal", "wood", "water", "fire" and "wind" with relation of mutual promotion and neutralization between them. During the long evolution, the meaning of the original five elements and their relationship were gradually amplified as they were applied into larger scope of phenomena and process in nature, society and human bodies. However, the rationale of all theories formed in those different fields, still clings to the specific properties of those five elements. Obviously such methods of thinking as analogy, metaphor and symbolism are working in the formation and development of these theories.

Another example is that all the five classics of Confucianism used image comparison to explain the theories and principles, especially Yi Jing and The Analects of Confucius. The character "Tao" is also a vague word connected to many concrete images and meanings, as road, path, or doctrine, truth, principle, law, or to thank, apologize, tell, explain, inform and so on. All these reflect the Chinese's inclination for imagery thinking or intuitional thinking.

\section{Synthetic thought pattern}

Synthetic thought pattern is characterized by its emphasis on the integrity of the society and nature. It regards man, nature, individual and society as an inalienable, mutual, influential organic whole and uses dialectic means to understand the harmony of the diversification and unity of the opposites. What the traditional Chinese philosophy seeks is the harmony of man and nature and the integrity of the heaven and man. Traces of this type of thinking could be dated back to the Zhou 
Dynasty when the Book of Change came into being. In this book, the initial schemata for holistic theory are drawn up. Similarly, Confucianism sees individual human being as an integral part of his environment and its main concern is to achieve interpersonal harmony in family and society. According to ancient Chinese literature, on the one hand, yin and yang are the opposing sides of a whole, but on the other hand, they exist independently. The loss of either of them would result in imbalance of the whole. Such holistic and harmonious thought patterns lead to the stress on balance, such as the equilibrium between man and nature.

Besides, this thought pattern is typically represented by traditional Chinese medicine and Peking Opera. According to traditional Chinese medicine, every part of the organs is connected with each other. Doctors usually take the whole body into consideration rather than only the condition of certain organs. Peking Opera also reflects the characteristics of this thought pattern. It is a comprehensive performance, and mixes many artistic forms together, such as singing, reading, and dancing.

\section{Research method and data collection}

\section{Research method}

This paper is a corpus-based study on the influence of Chinese thought patterns on English study. " a corpus is a collection of materials that has been made for particular purpose, such as a set of textbooks which are being analyzed and compared or a sample of sentences or utterances which are being analyzed for their linguistic features” (Richards, J.C., Platt \& H. Platt, 2000). “ It is a new technology in the study of language, which depends greatly on the use of computer.” (Dash,2005)

CLEC ( Chinese Learner English Corpus) is the biggest learners' corpus so far in China. Based on corpus linguistics and computer science, Chinese Learner English Corpus(CLEC) is selected as the target corpus. The CLEC is actually a collection of English compositions of Chinese learners at different stages ranging from intermediate and advanced university and college English majors and non-majors to secondary school students. It consists of five sub-corpora: middle school students (St2), college sophomores (St3) (Band IV students), college juniors (St4) (Band VI students), English major juniors (St5) and English major seniors (St6). The sampling involves a diversified and varied coverage of both student essays written on tests and different assignments completed in various other learning situations so that the corpus covers learners' written output widely enough. All the essays in the corpus are narrative and argumentative, amounting to 1 million words.

\section{The object of data collection}

The major feature of CLEC is that the learners' errors have been tagged within the corpus. For instance, the errors in spelling, phrases, lexicon, collocation and so on. They all have their own special tagging to represent themselves so that it is fast and convenient to concordance. For instance, at the lexical type of error, wd1 represent the errors in word order, wd2 the errors in part of speech, wd3 the errors in substitution and so on. As there are altogether more than sixty tagged errors which can be regarded as the influence of Chinese thought patterns on English study in CLEC, it is impossible to collect all the data owing to the limited space and time. Therefore, only a few errors are collected when they are used in discussing. In addition, due to the immature writing of middle school students and the limited number of students in English majors, most of the data are collected from St3 and St4 which are assumed to be more representative when discussing the problem of influence.

\section{Results and Discussion}

\section{Paratactic Preference}

Chinese tend to think intuitionally, which means that they appreciate the value of right brain, of intuition by which they can feel what they deal with. In other words, it means grasping things unconsciously or subconsciously by using feelings. In addition, as is discussed that the Chinese tend to think synthetically which means that they emphasize the harmony of man and nature and the integrity of heaven and man. Specifically, they pursue the unity and harmony of the "part" and the 
"whole", requiring understanding of things as a whole, thus the synthetic thought pattern is also called the holistic thought pattern. It employs synthesis in observing things and stresses intuition in comprehension. Consequently, it develops the emphasis on sense and meaning and at the same time neglects the formal logic, which reflects in Chinese language the paratactic preference, namely, valuing the integrity and completeness in content and meaning. According to the American Heritage Dictionary, parataxis refers to a language which word and sentence units are composed by means of their semantic coherence with diffusive linguistic forms and covert grammar.

\section{Parataxis at Lexical level}

The Paratactic preference in English learning is manifested at many aspects of lexical level such as word order which means the misplacement of any word; absence which means the omission of a word; substitution which means the error in word choice. Here, two typical examples of parataxis at lexical level will be discussed. They are redundancy which means the oversuppliance of a word and repetition which means the unnecessary repeating of a word. Redundancy is tagged as wd5 and repetition as wd6 in CLEC.

The redundant words can be nouns, verbs, prepositions or other parts in a sentence. Some of the errors are made due to the low proficiency level of students and their carelessness. However, some errors as redundant nouns are not so obvious if we don't check it carefully. The reasons for such errors are assumed to connect with the negative influence of Chinese thought patterns. Therefore, redundant nouns are discussed in detail. The followings are some examples of redundant nouns in st3 and st4:

[1] the mark is important, but the practice [ wd5 1-] experience is also necessary.

[2] work, and it is difficult for newly-graduates [ wd5 -1] men to find jobs.

[3] will have good luck in the future [ wd5 -1]days. [sn8,s] Now I live in the

[4] such as having difficulty in listening English [ wd5 4-] , doing experiments

It can be easily seen from these examples that there is a redundant noun in each sentence with the redundant one in bold. These nouns are unnecessary because their sense is already included or implied in some other element of the sentence. It is the result of the influence of the intuitional and synthetic thought pattern, or paratactic thought pattern when it is concerned with language in which the integrity and completeness in content and meaning is emphasized.

\section{Parataxis at Syntactical level}

Paratactic preference due to the influence of the intuitional and synthetic thought patterns can be seen at syntactic level. As is mentioned that paratactic language tends to apply semantic connection rather than syntactic devices to make clear the meaning of the speakers. As long as the meaning is delivered, there is no need to use cohesive devices like conjunctions. Logical connection is implicit rather than clearly indicated like English in sentence-arranging and idea-conveying process. As a result, the clause order of Chinese sentences is in line with the flow of thoughts. The meaning of a sentence changes as the clause order varies. Such a thought-oriented clause arrangement pattern is called the "flowing-water style" in which the grammatical relations of the language are usually to be sensed rather than explained by readers or listeners. Such characteristic of Chinese contributes to the run-on sentences which is tagged as sn1 in CLEC. It means the improper addition of clauses or fused sentence. Specifically, such sentences are formed by several clauses without connectors between them but only commas. For instance, the sentence "I came, I saw, I conquered." is a typical run-on sentence. Examples are as follows:

[1] efforts to play, but at last our class lose the match, but we still got the second place. [ sn1 s] This match [wd4,s]impressing me very much.

[2] and saw that the necklace was not around her neck any more, they couldn't find it, it was lost.[ sn1 s] They had to borrowed [vp5, 1-4] the money and bought the necklace to return Jeanne. During the

[3] soon. At last, people and fire fighters thanks to Liao Min's help, [sn8,s]no one was burnt by the fire.[ sn1 s]I live in Guang Dong Province with its capital Guang Zhou. [sn8,s] It lies in the south of

As can be seen from these examples,Students tend to apply synthetic and intuitional thought 
patterns when writing sentences. Under the influence of these thought patterns, the "flowing-water style” are obvious in English learning. Less grammatical connectors are used to organize the sentences and semantic connection is emphasized.

\section{Dynamic Preference}

As is discussed that synthetic though pattern emphasizes relation and connection, trying to keep the balance from the movement. In traditional Chinese culture, the universe was considered as a unity with perpetual transformation, a constant process of unceasing production and reproduction. Therefore, the consideration of everything in the world as fluid, ever changing and impermanent is emphasized. Thus, things are always required to be narrated as vividly as possible in Chinese, no matter whether they are outer events or inner psychological process, and in the narration, such descriptive words as adjectives and verbs are employed in large quantities. Therefore, in English learning, more verbs, adjectives and less prepositions are applied. For instance, the same meaning can be expressed by the second sentence through applying more nouns:

[1] The mayor said that he was very grateful because the construction workers were very dedicated and competent.

The mayor expressed his appreciation of the dedication and competence of the construction workers.

[2] If we don’t recognize serious the problem of growing population, we will make a bigmistake.

Failure to recognize the seriousness of growing population will lead to a big mistake.

After the analysis, it is not so hard to explain why the English beginners of Chinese fail to make such grammatically correct sentences as is shown in these examples collected form CLEC:

[1] because most of them are [ wd5 1-] contain too much flower, fat

[2] the first time, mathes [fm1,-] was [ wd5 1-3] became so interesting

[3] they are [ wd5 2-6] usually answer me carefully

The influence of Chinese thought patterns can be seen from these examples that verbs are piled up or used together to express ideas within a sentence, which is just against the English grammatical rules that there must be one predicate in a sentence.

\section{The Preference for the Topic Prominent Structure}

Influenced by the intuitional and synthetic thought patterns of the Chinese, the structure of Chinese is inevitably loose.It is well known that there are five basic sentence structures in English: SV, SVO, SVC, SVO1O2 and SVOC, each of which contains subject and predicate. It has become an invariable custom to have a subject before the predicate, and a sentence that does not contain a subject is felt to be incomplete. So if there is a predicate but no subject in a sentence, then a subject is assumed. The word "it" often suffices for the missing subject, as in the sentence: "It is 3 o'clock." On the contrary, the omission of the subjects is very common in Chinese. A Chinese sentence may have no definite subjects but it does have a topic at the begging, which together with the other components, constitutes “topic-prominence” sentences. But these topics don't necessarily serve as the subjects. For example:

[1] "Gong zuo de shi qing, ni ying gai kao lv xia”

Topic comment

[2] "Mei hua cheng shi, ren ren you ze"

\section{Topic comment}

In English, probably the most typical kind of sentence is the declarative which is made up of a subject, verb and direct object. Thus, English is assumed to be "subjet-prominent". The theory of subject-prominent and topic-prominent is established in $\mathrm{Li}$ and Thompson's paper "Subject and Topic: a New Typology of Language"(Li and Thompson, 1976). In this paper they hold that "many structural phenomena of a language can be explained on the basis of whether the basic structure of its sentences is analyzed as subject-predicate or topic-comment”. Thus, topic preference is assumed to be another influence of Chinese thought patterns on English learning. Evidence of it can be seen from the English writings of the Chinese students. In CLEC, topic-prominent sentence is tagged as sn5, examples of topic-prominent sentences are listed as follows:

[1] Getting to know the world outside the campus there are many ways. [ sn5 s-] Such as getting 
a part-time job when we

[2] Rain-water [fm2,-] river-water [fm2,-] and well-water [fm2,-] etc, all these are Fresh Water. [ sn5 s-] This is true.

[3] From the factories, [ sn5 -] the dirty water flows into the rivers and sea, the fishes [fm2,-] and other animals

It can be seen form these examples, it is hard for students to form a clear division line between subjects and predicates. Most of these sentences begins with a topic, the meaning of which is ambiguous and weird when considering from the point view of the native English speakers.

\section{Conclusion}

Nowadays, with the rapid development of China, the communication and contact between China and many other countries have grown steadily closer in the past decades in such aspects as economy, culture, and politics, etc. Foreign languages, English in particular, have being used frequently on many different occasions. Therefore, English learning is becoming more and more important among the Chinese. Then what are the key factors that influence the mastery of English has become the top issue to handle. The study on the influences of the Chinese thought patterns on English learning is beneficial for English learners to realize, overcome and take advantage of the negative influence of Chinese thought patterns on English learning and adopt a positive attitude towards the difficulties met in the process of language learning.

\section{References}

[1] Dash, N.S. Corpus linguistics and language technology: With reference to Indian language[M].New Delhi: Mittal Publications, 2005.

[2] Li , C. \& Thompson, S. Subject and Topic: A New Typology of Language [A]. In Li, C.(ed.). Subject and Topic [C]. New York: Academic Press, 1976.

[3]Richards, J.C., Platt. J. and Platt .H. Longman Dictionary of Language Teaching \& Applied Linguistics[M].Beijing: Foreign Language Teaching and Research Press, 2000. 